\title{
Monastrol analogs: A synthesis of pyrazolopyridine, benzopyranopyrazolopyri- dine, and oxygen-bridged azolopyrimidine derivatives and their biologicall screening
}

\author{
Jan Svetlik ${ }^{\mathrm{a}, *}$, Lucia Veizerováa ${ }^{\mathrm{a}}$, Thomas U. Mayer ${ }^{\mathrm{b}}$, Mario Catarinella ${ }^{\mathrm{b}}$ \\ ${ }^{a}$ Department of Pharmaceutical Analysis and Nuclear Pharmacy, Comenius University, Odbojarov 10, SK-832 32 Bratislava, Slovak Republic \\ bepartment of Molecular Genetics, University of Konstanz, 78457 Konstanz, Germany
}

\section{A R T I C L E I N F O}

\section{Article history:}

Received 14 April 2010

Revised 18 May 2010

Accepted 19 May 2010

Available online 26 May 2010

\section{Keywords:}

Biginelli reaction

Hantzsch reaction

oxygen-bridged nitrogen heterocycles

Monastrol analogs

\begin{abstract}
A B S T R A C T
A synthesis of novel pyrazolopyridine, benzopyranopyrazolopyridine, and oxygen-bridged pyrazolo-, tetrazolo-, benzimidazo-, and thiazolopyrimidines via Hantzsch- and Biginelli-like condensations has been developed. The ability of these compounds to inhibit Eg5 activity has been examined. The results indicate that synthetic manipulations in the monastrol thiourea moiety are inefficient.
\end{abstract}

Monastrol ( \pm )-1 (Fig. 1) is a simple low molecular weight molecule that was discovered a decade ago to be a cell-permeable kinesin Eg5 inhibitor ${ }^{1}$; this discovery initiated a further stage in Biginelli 3,4-dihydropyrimidine-2(1H)-one chemistry. The new era in the long time history of this heterocyclic family ${ }^{2}$ has been started in Squibb laboratories by detailed investigations ${ }^{3}$ of its earlier recognized antihypertensive properties. In addition to the targeted $\mathrm{Ca}^{2+}$ channel blockers, research was independently set off that was oriented toward dihydropyrimidine selective $\alpha_{1 \mathrm{~A}}$ adrenergic receptor antagonists for treatment of benign prostatic hyperplasia. ${ }^{3}$ Convergently, the close resemblance to the 1,4-dihydropyridine core plays a crucial role in both goals. 3,4-Dihydropyrimidinone-5-carboxylate scaffold has also emerged as an integral backbone of some neuropeptide Y (NPY) antagonists. ${ }^{3}$ Furthermore, recent pharmacological evaluations of Biginelli compounds disclosed that these derivatives also functioned as melanin concentrating hormone receptor (MCH1-R) antagonists, ${ }^{4}$ chemical modulators of heat shock protein 70 ( Hsp 70), hepatitis B replication inhibitors, ${ }^{6}$ and inhibitors of the fatty acid transporters. ${ }^{7}$ In particular, a simple 4-aryldihydropyrimidine derivative was recently discovered to be highly effective against methicillin-resistant strain of Staphylococcus aureus (MRSA). ${ }^{8}$

In recent years, a significant progress has been reported in the new class of promising anticancer agents led by monastrol 1 .

* Corresponding author.

E-mail address: svetlik@fpharm.uniba.sk (J. Svetlik).
Diverse structures that effectively inhibit bipolar mitotic spindle assembly have been found. ${ }^{9}$ Abiding interest in compound $\mathbf{1}$ has led to conformative skeleton modifications of the parent ring by annelation across the $\mathrm{C}-5-\mathrm{C}-6$ bond to provide more potent ana$\operatorname{logs},{ }^{10}$ such as dimethylenastron 2 and quinazoline- $2(1 \mathrm{H})$-thione 3 (Fig. 1). Considerable work has also been devoted to gaining insight into the structure-activity relationship in the monastrol derivative series. ${ }^{11}$ However, C-6 substitution has not been explored. In order to probe the effect of methyl functionalization in position 6 on the ability to inhibit Eg5 activity, we have recently synthesized analogous Biginelli compounds 4 bearing methyl acetate unit at C-6. ${ }^{12}$ The conformationally restricted dihydropyrimidine prototype ${ }^{12} 5$ completed our trial set (Fig. 2). Some of screened samples exhibited in vitro inhibitory effect at concentrations of $100-200 \mu \mathrm{M}$ in semi-quantitative assays; summing up the<smiles>CCOC(=O)C1=C(C)NC(=[SH]C2(C)CC(=O)C3=C(C2)NC(=S)NC3c2cccc(O)c2)NC1c1cccc(O)c1</smiles>

1 2

Figure 1. 
<smiles>[X]C1=NC(c2ccccc2)C(C(=O)OC)=C(CCOC)N1</smiles><smiles>[X]C1=N[C@]2(CCOC)O[C@]3(C(C)=O)c4ccccc4[C@H]2[C@@]3(C)N1</smiles>

4
Figure 2.

testing, pyrimidine preparates were at least 10 times less active than the monastrol standard. ${ }^{13}$

Based on the reported remarkable data for simple pyrazoles as kinesin spindle protein (KSP) inhibitors, ${ }^{9 a}$ we directed our studies at monastrol-related fused heteroanalogs. As a structural modification we chose to integrate the monastrol key building block, pyrimidine nucleus, and the five-membered nitrogen ring system into a common, single heterocyclic molecule. Here we wish to describe the synthesis of novel condensed azolopyridines and azolopyrimidines of pharmacological interest. The biological evaluation of the prepared derivatives toward inhibition of KSP enzymatic activity is also briefly described.

First, we examined a three-component reaction of 3-hydroxybenzaldehyde 6 with methyl acetoacetate 7 and 5-amino-3methyl-1H-pyrazole 8 adopting Quiroga's protocol. ${ }^{14}$ Refluxing the reactants in EtOH for $1.5 \mathrm{~h}$ afforded product 9 whose ${ }^{1} \mathrm{H}$ NMR spectrum lacked characteristic ${ }^{14 b, 15}$ singlet for the pyrazole methine near $6.0 \mathrm{ppm}$. This revealed a cyclization mode leading to pyrazolopyridine 9 and ruled out the pyrazolopyrimidine alternative $\mathbf{9}^{\prime}$ (Scheme 1). A similar behavior of the aminopyrazole has been already reported. ${ }^{14}$
However, when salicylaldehyde was employed, a bicyclic annelation accompanied by lactonization took place to yield tetracyclic product 11. A small quantity of oxidized form 12 in a second crop indicated that 1,4-dihydropyridine ring was partially oxidized in air. Therefore, the reaction was carried out under nitrogen which decreased the 12:11 ratio to $1: 24$ (Scheme 2).

To exploit the synthetic potential of amine 8 , we used our methodology ${ }^{16}$ based upon 4-(2-hydroxyphenyl)but-3-en-2-one 13 for the preparation of conformationally restricted heterocycles having the aromatic ring fixed by an oxygen-bridge. In this case, harsher reaction conditions were necessary (reflux in DMF, $1 \mathrm{~h}$ ) to achieve heterocyclization ( $\rightarrow \mathbf{1 4}$, Scheme 3 ). Apparently, of the three nucleophilic nitrogen centers capable of attacking the olefinic enone $\beta$-carbon, the $\mathrm{sp}^{3} \mathrm{~N}-1$ atom of pyrazole tautomer 8 triggers the multistep sequence. Michael addition forms the pyrazolopyrimidine skeleton which is followed by subsequent O-bridge formation. Detailed ${ }^{1} \mathrm{H}$ and ${ }^{13} \mathrm{C}$ NMR analysis was performed using 2D-correlation techniques (COSY, HSQC, and HMBC). Moreover, close spatial proximities for 5-Me- $\mathrm{NH}$ and $\mathrm{NH}-$ pyrazole- $\mathrm{H}$, established from 1D-NOESY measurements, clearly pointed to a linear arrangement of both nitrogen rings in tetracyclic molecule 14. An interesting feature was found in the aliphatic region of the ${ }^{1} \mathrm{H}$ NMR spectrum. The $\mathrm{CH}_{2} \mathrm{CH}$ moiety and $\mathrm{NH}$ constitute a four spin system (ABMX) as a result due to a planar long-range coupling of the $\mathrm{W}$ type between the amine and equatorial diastereotopic methylene protons. Accordingly, one of the two $\mathrm{CH}_{2}$ signals which appears at a higher field as a doublet with a fine triplet splitting pattern could be assigned to $\mathrm{H}-13$ equiv.

To further extend our set for activity screening, we employed some other heterocyclic amines for the above cyclocondensation. Upon refluxing enone template $\mathbf{1 3}$ with an equimolar amount of 5-amino- $1 \mathrm{H}$-tetrazole monohydrate in DMF for $2 \mathrm{~h}$ the expected polyazaheterocycle $\mathbf{1 5}$ was obtained. Likewise, treatment of<smiles>O=Cc1cccc(O)c1</smiles>

6<smiles>CCCCC(C)OC</smiles>

7<smiles>Cc1cc(N)[nH]n1</smiles>

8

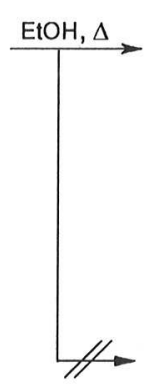<smiles>CC(=O)C1=C(C)Nc2cc(C)nn2C1c1cccc(O)c1</smiles><smiles>CC(=O)C1=C(C)Nc2n[nH]c(C)c2C1c1cccc(O)c1</smiles>
9

Scheme 1.<smiles>O=Cc1ccccc1O</smiles><smiles>CC1=C2C(=O)Oc3ccccc3[C@@H]2c2c(n[nH]c2C)N1</smiles>

11 
<smiles>CC(=O)/C=C/c1ccccc1Cl</smiles>

13

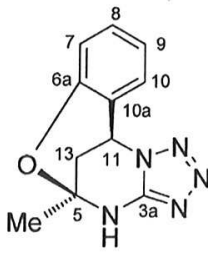

15

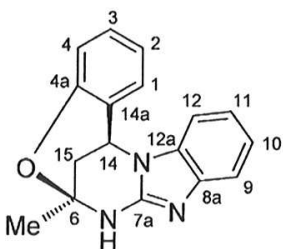

16

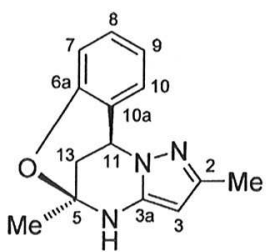

14
Scheme 3.

Figure 3.

butenone 13 with 2-aminobenzimidazole gave rise to pentacyclic product 16 in a good yield (Fig. 3). Full assignment of the ${ }^{1} \mathrm{H}$ and ${ }^{13} \mathrm{C}$ NMR signals for 16 has been established and additional NMR data concerning benzimidazole chemical shifts were invoked ${ }^{17}$ to support our analysis. The oxygen-bridged fused ring system with nitrogen at the bridgehead position (16) represents, to our knowledge, a new class of condensed heterocyclic compounds. Although pyrazolo- and tetrazolobenzoxadiazocine heterocyclic families such as 14 and $\mathbf{1 5}$ have been described, ${ }^{18}$ our approach has opened an alternative route to these derivatives. Finally, thiazolobenzoxadiazocine $\mathbf{1 7}$ possessing a thiourea structural motif was likewise synthesized using 2-amino-2-thiazoline to enlarge the tested group (Fig. 3). It should be noted that a hydrobromide salt of $\mathbf{1 7}$ was already prepared by us via cyclization of an oxygenbridged thioxopyrimidine with 1,2-dibromoethane. ${ }^{19}$ Furthermore, we recently described a single-crystal X-ray analysis of $17 .^{20}$

All the compounds prepared here ${ }^{21}$ as monastrol mimics were tested for their ability to inhibit KSP enzymatic activity by using an in vitro ATPase assay and an in vivo cell-based assay inducing mitotic arrest in HeLa cells according to a method described in Ref. 10b. Unfortunately, it followed from the biological screening that none of the investigated compounds has shown any significant inhibitory effect in vitro or monoastral spindle phenotype in vivo. Moreover, benzimidazole derivative $\mathbf{1 6}$ appears to be toxic to BSC-1 and HeLa cell lines at $100 \mu \mathrm{M}$. It can be concluded that structural modification of monastrol in the thiourea moiety by fusion with additional rings results in a pronounced negative effect on the inhibition potency against KSP. In view of these findings, annelation of parent monastrol on left-hand side as reported by Mayer and Giannis proved to be a rational step in further development of effective analogs. ${ }^{10}$

In summary, we described syntheses of novel monastrol analogs based on Hantzsch- and Biginelli-like reactions. The new series consisted of pyridine and pyrimidine derivatives modified by fusion with azoles and also by additional conformative bridging through the phenol oxygen atom. Although the new compounds have been found to be inactive against Eg5 mitotic kinesin, the reported results contribute toward deeper insight into structureactivity relationship.

\section{Acknowledgments}

This work was supported by a Grant of Pharmaceutical Faculty (\#FaF UK/14/2009). The NMR experimental part of this work was facilitated by the support of Slovak National Research and Development Program No. 2003SP200280203.

\section{References and notes}

1. Mayer, T. U.; Kapoor, T. M.; Haggarty, S. J.; King, R. W.; Schreiber, S. L.; Mitchison, T. J. Sci. 1999, 286, 971.

2. (a) Biginelli, P. Gazz. Chim. Ital. 1893, 23, 360; (b) Kappe, C. O. Acc. Chem. Res. 2000, 33, 879 .

3. Kappe, C. O. Eur. J. Med. Chem. 2000, 35, 1043. and references cited therein.

4. Basso, A. M.; Bratcher, N. A.; Gallagher, K. B.; Cowart, M. D.; Zhao, C.; Sun, M.; Esbenshade, T. A.; Brune, M. E.; Fox, G. B.; Schmidt, M.; Collins, C. A.; Souers, A. J.: Iyengar, R.; Vasudevan, A.; Kym, P. R.; Hancock, A. A.; Rueter, L. E. Eur. J. Pharmacol. 2006, 540, 115.

5. Wisen, S.; Androsavich, J.; Evans, C. G.; Chang, L.; Gestwicki, J. E. Bioorg. Med. Chem. Lett. 2008, 18, 60

6. Deres, K.; Schröder, C. H.; Paessens, A.; Goldmann, S.; Hacker, H. J.; Weber, O.; Krämer, T.: Niewöhner, U.: Pleiss, U.: Stoltefuss, J.: Graef, E.; Koletzki, D.; Masantschek, R. N. A.; Reimann, A.; Jaeger, R.; Groß, R.; Beckermann, B.; Schlemmer, K.-H.; Haebich, D.; Rübsamen-Wagmann, H. Science 2003, 299, 893.

7. Blackburn, C.; Guan, B.; Brown, J.; Cullis, C.; Condon, S. M.: Jenkins, T. J.; Peluso, S.; Ye, Y.; Gimeno, R. E.; Punreddy, S.; Sun, Y.; Wu, H.; Hubbard, B.; Kaushik, V. Tummino, P.; Sanchetti, P.; Sun, D. Y.; Daniels, T.; Tozzo, E.; Balani, S. K.; Raman, P. Bioorg. Med. Chem. Lett. 2006, 16, 3504.

8. Wyatt, E. E.; Galloway, W. R. J. D.; Thomas, G. L.; Welch, M.; Loiseleur, O.; Plowright, A. T.; Spring, D. R. Chem. Commun. 2008, 4962.

9. (a) Cox, C. D.; Breslin, M. J.; Whitman, D. B.; Coleman, P. J.; Garbaccio, R. M.; Fraley, M. E.; Zrada, M. M.; Buser, C. A.; Walsh, E. S.; Hamilton, K.; Lobell, R. B.; Tao, W. Abrams, M. T. South, V. J. Huber, H. E. Kohl, N. E. Harman, G.; Bioorg. Med. Chem. Lett. 2007, 17, 2697; (b) DeBonis, S.; Skoufias, D. A.; Indorato, R.-L.; Liger, F.; Marquet, B.; Laggner, C.; Benoît, J.; Kozielski, F. J. Med. Chem. 2008, 51, 1115; (c) Tarby, C. M.; Kaltenbach, R. F.; Huynh, T.; Pudzianowski, A.; Shen, H.; Ortega-Nanos, M.; Sheriff, S.; Newitt, J. A.; McDonell, P. A.; Burford, N.; Fairchild, C. R.; Vaccaro, W.; Chen, Z.; Borzilleri, R. M.; Naglich, J.; Lombardo, L. J.: Gottardis, M.: Trainor, G. L.: Roussell, D. L. Bioorg. Med. Chem. Lett. 2006, 16 , 2095; (d) Sunder-Plassmann, N.; Sarli, V.; Gartner, M.; Utz, M.; Seiler, J.: Huemmer, S.; Mayer, T. U.; Surrey, T.; Giannis, A. Bioorg. Med. Chem. 2005, 13, 6094; (e) Kim, K. S.; Lu, S.; Cornelius, L. A.; Lombardo, L. J.; Borzilleri, R. M.; Schroeder, G. M.; Sheng, C.; Rovnyak, G.; Crews, D.; Schmidt, R. J.; Williams, D. K.; Bhide, R. S.; Traeger, S. C.; McDonnell, P. A.; Mueller, L.; Sheriff, S.; Newitt, J. A.; Pudzianowski, A. T.; Yang, Z.; Wild, R.; Lee, F. Y.; Batorsky, R.; Ryder, J. S.; Ortega-Nanos, M.; Shen, H.; Gottardis, M.; Roussell, D. L. Bioorg. Med. Chem. Lett. 2006, 16, 3937; (f) Sakowicz, R.; Finer, J. T.; Beraud, C.; Crompton, A.; Lewis, E.; Fritsch, A.; Lee, Y.; Mak, J.; Moody, R.; Turincio, R.; Chabala, J. C.; Gonzales, P.; Roth, S.; Weitman, S.; Wood, K. V. Cancer Res. 2004, 64, 3276; (g) Matsuno, K.; Sawada, J.; Sugimoto, M.; Ogo, N.; Asai, A. Bioorg. Med. Chem. Lett. 2009, 19. 1058.

10. (a) Gartner, M.: Sunder-Plassmann, N.: Seiler, J.; Utz, M.; Vernos, I.; Surrey, T. Giannis, A. ChemBioChem 2005, 6, 1173; (b) Sarli, V.: Huemmer, S.: SunderPlassmann, N.; Mayer, T. U.; Giannis, A. ChemBioChem 2005, 6, 2005.

11. Klein, E.; DeBonis, S.; Thiede, B.; Skoufias, D. A.; Kozielski, F.; Lebeau, L. Bioorg Med. Chem. 2007, 15, 6474

12. Svetlik, J.; Veizerova, L.; Kettmann, V. Tetrahedron Lett. 2008, 49, 3520.

13. Mayer, T. U. Unpublished results.

14. (a) Quiroga, J.; Insuasty, B.; Hormaza, A.; Saitz, C.; Jullian, C. J. Heterocycl. Chem 1998, 35, 575; (b) Quiroga, J.; Mejia, D.; Insuasty, B.; Abonia, R.; Nogueras, M.; Sanchez, A.; Cobo, J.; Low, J. N. Tetrahedron 2001, 57, 6947.

15. Quiroga, J.; Insuasty, B.; Rincon, R.; Larrahondo, M.; Hanold, N.; Meier, H. J. Heterocycl. Chem. 1994, 31, 1333.

16. (a) Svetlik, J.; Turecek, F.; Hanus, V. J. Chem. Soc., Perkin Trans. 1 1988, 2053; (b) Svetlik, J.; Hanus, V.; Bella, J. Liebigs Ann. Chem. 1989, 91; (c) Svetlik, J.; Turecek, F.: Hanus, V. J. Chem. Soc., Perkin Trans. 1 1990, 1315.

17. (a) Papadopoulos, E. P.; Hollstein, U. Org. Magn. Reson. 1982, 19, 188; (b) Lopyrev, V. A.; Larina, L. I.; Vakulskaya, T. I.; Shibanova, E. F.; Titova, I. A. Voronkov, M. G.; Liepinsh. E. E. Org. Magn. Reson. 1982, 20, 212; (c) Claramunt, R. M.; Elguero, J.; Meco, T. J. Heterocycl. Chem. 1983, 20, 1245.

18. Bartashevich, E. V.; Plekhanov, P. V.; Rusinov, G. L.; Potemkin, V. A.; Belik, A. V.; Chupakhin, O. N. Izv. Akad. Nauk SSSR Ser. Khim. 1999, 1573.

19. Svetlik, J.; Hanus, V.; Bella, J. J. Chem. Res., Synop. 1991, 4.

20. Kettmann, V.; Svetlik, J.; Veizerova, L. Acta Crystallogr., Sect. E 2009, 65, 2967.

21. Methyl 4-(3-hydroxyphenyl)-3,6-dimethyl-4,7-dihydro-2H-pyrazolo[3,4-b]pyridine-5-carboxylate (9): $\mathrm{Mp} 268-269{ }^{\circ} \mathrm{C}(\mathrm{MeOH})$, yield 36\%; IR $(\mathrm{KBr}): y_{\mathrm{m}}$ $3372,3272,1667,1541,1508,1233,1101 \mathrm{~cm}^{-1} ;{ }^{1} \mathrm{H}$ NMR (DMSO- $\left.d_{6}\right) \delta 1.91(\mathrm{~s}$ $3 \mathrm{H}, \mathrm{Me}-3), 2.31$ (s, 3H, Me-6), 3.41 (s, 3H, OMe), 4.87 (s, 1H, H-4), 6.45 (dd, $1 \mathrm{H}$ $J=7.8$ and $\left.1.8 \mathrm{~Hz}, \mathrm{H}-4^{\prime}\right), 6.50\left(\mathrm{~s}, 1 \mathrm{H}, \mathrm{H}-2^{\prime}\right), 6.58\left(\mathrm{~d}, 1 \mathrm{H}, J=7.8 \mathrm{~Hz}, \mathrm{H}-\mathrm{G}^{\prime}\right), 6.97$ (t. $\left.\left.1 \mathrm{H}, J=7.8 \mathrm{~Hz}, \mathrm{H}-5^{\prime}\right), 9.10(\mathrm{~s}, 1 \mathrm{H}, \mathrm{OH} / \mathrm{NH}), 9.31(\mathrm{~s}, 1 \mathrm{H}, \mathrm{NH} / \mathrm{OH})\right), 11.63(\mathrm{~s}, 1 \mathrm{H}$ NH-2). 1,5-Dimethyl-4,1 1 b-dihydro-[1]benzopyrano[4,3-d]pyrazolo[3,4-b]pyridin-6(2H)-one (11): $\mathrm{Mp}>300^{\circ} \mathrm{C}(\mathrm{EtOH})$, yield $89 \%$; IR (KBr): $v_{\max } 3287,1695$ $1596,1546,1503,1199 \mathrm{~cm}^{-1}$ : ${ }^{H}$ H NMR (DMSO-d $\left.d_{6}\right) \delta 2.15(\mathrm{~s}, 3 \mathrm{H}, \mathrm{Me}-1), 2.24(\mathrm{~s}$ $3 \mathrm{H}, \mathrm{Me}-5), 4.86(\mathrm{~s}, 1 \mathrm{H}, \mathrm{H}-11 \mathrm{~b}), 6.94(\mathrm{~d}, 1 \mathrm{H}, J=7.5 \mathrm{~Hz}, \mathrm{H}-8), 7.06(\mathrm{~d}, 1 \mathrm{H}, J=7.5 \mathrm{~Hz}$, 
$\mathrm{H}-11), 7.10(\mathrm{t}, 1 \mathrm{H}, J=7.5 \mathrm{~Hz}, \mathrm{H}-10), 7.23(\mathrm{t}, 1 \mathrm{H}, J=7.5 \mathrm{~Hz}, \mathrm{H}-9), 9.76(\mathrm{~s}, 1 \mathrm{H}, \mathrm{NH})$ $12.10(\mathrm{~s}, 1 \mathrm{H}, \mathrm{NH}) .1,5$-Dimethyl-[1]benzopyrano[4,3-d]pyrazolo[3,4-b]pyridin 6(3H)-one (12): $\mathrm{Mp}>300{ }^{\circ} \mathrm{C}(\mathrm{EtOH})$, yield 4\%; $\mathrm{IR}(\mathrm{KBr}): v_{\max } 1735,1611,1542$ $1250 \mathrm{~cm}^{-1}$; ' $\mathrm{H}$ NMR (DMSO-d $\left.\mathrm{d}_{6}\right) \delta 2.78(\mathrm{~s}, 3 \mathrm{H}, \mathrm{Me}-1), 2.94(\mathrm{~s}, 3 \mathrm{H}, \mathrm{Me}-5), 7.44$ (d, $1 \mathrm{H}, J=7.8 \mathrm{~Hz}, \mathrm{H}-8), 7.48(\mathrm{t}, 1 \mathrm{H}, J=7.8 \mathrm{~Hz}, \mathrm{H}-10), 7.71(\mathrm{t}, 1 \mathrm{H}, J=7.8 \mathrm{~Hz}, \mathrm{H}-9), 8.36$ (d, $1 \mathrm{H}, J=7.8 \mathrm{~Hz}, \mathrm{H}-11$ ), 13.8 (br s, $1 \mathrm{H}, \mathrm{NH}$ ). (5R* $\left.11 R^{*}\right)-( \pm$ )-2,5-Dimethyl-4,5dihydro-11H-5,11-methanopyrazolo[5,1-d][1,3,5]benzoxadiazocine (14): Mp dihydro- $11 H-5,11-$ methanopyrazolo[5,1-d] [1,3,5]benzoxadiazocine (14): Mp
$208-210^{\circ} \mathrm{C}(\mathrm{MeCN})$, yield 43\%; IR ( $\left.\mathrm{KBr}\right): v_{\max } 3421,1609,1589,1578$, $208-210^{\circ} \mathrm{C}(\mathrm{MeCN})$, yield 43\%; IR ( $\left.\mathrm{KBr}\right): v_{\max } 3421,1609,1589,1578$,
$1461,1256,1068,843,753,729 \mathrm{~cm}^{-1} ;{ }^{1} \mathrm{H}$ NMR (DMSO-d $\left.d_{6}\right) \delta 1.71(\mathrm{~s}, 3 \mathrm{H}, \mathrm{Me}-5$ ), $1.94(\mathrm{~s}, 3 \mathrm{H}, \mathrm{Me}-2), 2.26$ (quasi dt, $1 \mathrm{H}, J=13.2 \mathrm{~Hz}, \mathrm{H}-13$ equiv), 2.38 (dd, $1 \mathrm{H}$ $J=13.2$ and $3.0 \mathrm{~Hz}, \mathrm{H}-13 \mathrm{ax}$ ), $4.97(\mathrm{~s}, 1 \mathrm{H}, \mathrm{H}-3), 5.17$ (quasi t, $1 \mathrm{H}, J=2.8 \mathrm{~Hz}, \mathrm{H}-11$ ), $6.74(\mathrm{dd}, 1 \mathrm{H}, J=8.4$ and $1.2 \mathrm{~Hz}, \mathrm{H}-7), 6.79(\mathrm{dt}, 1 \mathrm{H}, J=7.8$ and $1.8 \mathrm{~Hz}, \mathrm{H}-9$ ), 7.12 (dt, $1 \mathrm{H}, J=7.8$ and $1.8 \mathrm{~Hz}, \mathrm{H}-8), 7.25$ (dd, $1 \mathrm{H}, 1 \mathrm{H}, J=7.5$ and $1.2 \mathrm{~Hz}, \mathrm{H}-10), 7.50$ (s, $1 \mathrm{H}, \mathrm{NH}) ;{ }^{13} \mathrm{C}$ NMR (DMSO- $\left.d_{6}\right) \delta 13.8(\mathrm{Me}-2), 26.7(\mathrm{Me}-5), 32.5\left(\mathrm{CH}_{2}\right), 49.9(\mathrm{CH}$ $11), 81.9(\mathrm{C}-5), 85.2(\mathrm{CH}-3), 115.8(\mathrm{CH}-7), 119.5(\mathrm{CH}-9), 123.1$ (C-10a), $128.6(\mathrm{CH}$ 10), 129.1 (CH-8), 142.4 (C-3a), $146.3(\mathrm{C}-2), 153.8$ (C-6a); EI MS (m/z) $241\left(\mathrm{M}^{+}\right)$ $\left(5 R^{*}, 11 R^{*}\right)-( \pm)-5-M e t h y l-4,5-d i h y d r o-11 H-5,11-$ methanotetrazolo[5,1-d][1,3,5]benzoxadiazocine (15): $\mathrm{Mp} 231-233^{\circ} \mathrm{C}(\mathrm{MeOH})$, yield 34\%; IR (KBr): $v_{\max } 3233$ $1624,1485,1252,1113,1081,868,761 \mathrm{~cm}^{-1} ;{ }^{1} \mathrm{H}$ NMR (DMSO-d $\left.d_{6}\right) \delta 1.81(\mathrm{~s}, 3 \mathrm{H}$ Me), 2.48 (ddd, $1 \mathrm{H}, J=13.8,3.0$ and $1.2 \mathrm{~Hz}, \mathrm{H}-13$ equiv), 2.50 (dd, $1 \mathrm{H}, J=13.8$ and $3.0 \mathrm{~Hz}, \mathrm{H}-13 \mathrm{ax}), 6.01(\mathrm{t}, 1 \mathrm{H}, J=3.0 \mathrm{~Hz}, \mathrm{H}-11), 6.84(\mathrm{~d}, 1 \mathrm{H}, J=7.8 \mathrm{~Hz}, \mathrm{H}-7), 6.89(\mathrm{t}$ $1 \mathrm{H}, J=7.8 \mathrm{~Hz}, \mathrm{H}-9), 7.22(\mathrm{dt}, 1 \mathrm{H}, J=8.2$ and $1.8 \mathrm{~Hz}, \mathrm{H}-8), 7.39$ (dd, $1 \mathrm{H}, J=7.8$ and
$1.8 \mathrm{~Hz}, \mathrm{H}-10), 9.19(\mathrm{~s}, 1 \mathrm{H}, \mathrm{NH}):{ }^{13} \mathrm{C} \mathrm{NMR}$ (DMSO- $\left.d_{6}\right) \delta 25.7(\mathrm{Me}), 31.0\left(\mathrm{CH}_{2}\right), 48.5$ (CH-11), 82.4 (C-5), 116.6 (CH-7), 120.6 (CH-9), 120.8 (C-10a), 128.6 (CH-10) $130.3(\mathrm{CH}-8), 152.1$ (C-3a), 152.2 (C-6a); $\left(6 R^{*}, 14 R^{*}\right)-( \pm)-6-M e t h y l-6,7-d i h y d r o-$ 14H-6,14-methanobenzimidazo[2,1-d][1,3,5]benzoxadiazocine (16): Mp 264$266^{\circ} \mathrm{C}(\mathrm{EtOH})$, yield 64\%: IR (KBr): $v_{\max } 3215,1641,1604,1578,1460,1255$ $1189,1138,1073,754 \mathrm{~cm}^{-1}$; ${ }^{1} \mathrm{H}$ NMR (DMSO- $d_{6}$ ) $\delta 1.81$ (s, $3 \mathrm{H}, \mathrm{Me}$ ), 2.46 (dd, $1 \mathrm{H}$ $J=13.2$ and $2.4 \mathrm{~Hz}, \mathrm{H}-15$ equiv), 2.52 (dd, $1 \mathrm{H}, J=13.2$ and $3.6 \mathrm{~Hz}, \mathrm{H}-15 \mathrm{ax}$ ), 5.68 , (t, $1 \mathrm{H}, J=3.0 \mathrm{~Hz}, \mathrm{H}-14), 6.79(\mathrm{t}, 1 \mathrm{H}, J=7.2 \mathrm{~Hz}, \mathrm{H}-2), 6.80(\mathrm{~d}, 1 \mathrm{H}, J=7.9 \mathrm{~Hz}, \mathrm{H}-4)$ $6.96(\mathrm{~m}, 2 \mathrm{H}, \mathrm{H}-10+\mathrm{H}-11), 7.14(\mathrm{t}, 1 \mathrm{H}, J=7.2 \mathrm{~Hz}, \mathrm{H}-3), 7.15(\mathrm{~m}, 1 \mathrm{H}, \mathrm{H}-9), 7.45(\mathrm{~d}$ $1 \mathrm{H}, J=7.2 \mathrm{~Hz}, \mathrm{H}-1), 7.62(\mathrm{~m}, 1 \mathrm{H}, \mathrm{H}-12), 8.75$ (br s, $1 \mathrm{H}, \mathrm{NH}$ ); ${ }^{13} \mathrm{C}$ NMR (DMSO-d $\left.\mathrm{d}_{6}\right)$ 26.4 (Me), $31.8\left(\mathrm{CH}_{2}\right), 45.1$ ( $\left.\mathrm{CH}-14\right), 82.1$ (C-6), 107.9 ( $\left.\mathrm{CH}-12\right), 115.4(\mathrm{CH}-9)$ $116.3(\mathrm{CH}-4), 118.6(\mathrm{CH}-11), 119.9(\mathrm{CH}-2), 120.8(\mathrm{CH}-10), 122.5(\mathrm{C}-14 \mathrm{a}), 128.5$ $(\mathrm{CH}-1), 129.5(\mathrm{CH}-3), 131.7(\mathrm{C}-12 \mathrm{a}), 142.7$ (C-8a), 150.4 (C-7a) $152.3(\mathrm{C}-4 \mathrm{a})$ $\left(5 R^{*}, 11 R^{*}\right)-( \pm)-5$-Methyl-1,2-dihydro-5H,11H-5,11-methano[1,3]thiazolo[2,3-d]$1,3,5$ ]benzoxadiazocine (17): $\mathrm{Mp} 240-241{ }^{\circ} \mathrm{C}(\mathrm{MeCN})$, yield 64\%; IR (KBr): $v_{\max }$ $1588,1481,1253,1174,1139,879,762 \mathrm{~cm}^{-1},{ }^{1} \mathrm{H}$ NMR (DMSO-d $\left.d_{6}\right) \delta 1.55(\mathrm{~s}, 3 \mathrm{H}$ Me), 2.07 (dd, $1 \mathrm{H}, J=13.2$ and $3.0 \mathrm{~Hz}, \mathrm{H}-13), 2.14$ (dd, $1 \mathrm{H}, J=13.2$ and $3.0 \mathrm{~Hz}, \mathrm{H}-$ 13'), $3.08(\mathrm{~m}, 1 \mathrm{H}, \mathrm{H}-2), 3.18\left(\mathrm{~m}, 2 \mathrm{H}, \mathrm{H}-2^{\prime}+\mathrm{H}-1\right), 3.72\left(\mathrm{~m}, 1 \mathrm{H}, \mathrm{H}-1^{\prime}\right), 4.47(\mathrm{t}, 1 \mathrm{H}$ $J=3.0 \mathrm{~Hz}, \mathrm{H}-11), 6.79(\mathrm{~d}, 1 \mathrm{H}, J=7.2 \mathrm{~Hz}, \mathrm{H}-7), 6.85(\mathrm{t}, J=7.2 \mathrm{~Hz}, \mathrm{H}-9), 7.19(\mathrm{~m}, 2 \mathrm{H}$ $\mathrm{H}-8+\mathrm{H}-10) ;{ }^{13} \mathrm{C}$ NMR (DMSO-d $\left.\mathrm{d}_{6}\right) \delta 26.0\left(\mathrm{CH}_{2}-2\right), 28.4(\mathrm{Me}), 31.2\left(\mathrm{CH}_{2}-13\right), 49.1$ $(\mathrm{CH}-11), 51.1\left(\mathrm{CH}_{2}-1\right), 82.9(\mathrm{C}-5), 116.4(\mathrm{CH}-7), 119.3(\mathrm{CH}-9), 120.6(\mathrm{C}-10 \mathrm{a})$ 129.4 (CH-10), 129.5 (CH-8), 152.5 (C-6a), 164.6 (C-3a) 